\title{
The nonsense-mediated mRNA decay (NMD) pathway differentially regulates COX17, COX19 and COX23 mRNAs
}

\author{
Kaitlin Murtha ${ }^{1} \cdot$ Munok Hwang ${ }^{1} \cdot$ Megan C. Peccarelli $^{1} \cdot$ Taylor D. Scott $^{2} \cdot$ Bessie W. Kebaara $^{1} \mathbb{C}$
}

Received: 3 July 2018 / Revised: 3 October 2018 / Accepted: 5 October 2018 / Published online: 13 October 2018

(C) The Author(s) 2018

\begin{abstract}
The differential regulation of COX17, COX19 and COX23 mRNAs by the nonsense-mediated mRNA decay (NMD) pathway was investigated. The NMD pathway regulates mRNAs that aberrantly terminate translation. This includes mRNAs harboring premature translation termination codons and natural mRNAs. Most natural mRNAs regulated by NMD encode fully functional proteins involved in various cellular processes. However, the cause and targeting of most of these mRNAs by the pathway is not understood. Analysis of a set of mRNAs involved in copper homeostasis showed that a subset of these mRNAs function in mitochondrial copper homeostasis. Here, we examined the regulation of COX17, COX19 and COX23 mRNAs by NMD. These mRNAs encode homologous mitochondrial proteins involved in metallation of cytochrome $c$ oxidase. We found that COX17, COX19 and COX23 mRNAs are differentially regulated by NMD depending on environmental copper levels. A long 3'-UTR contributes to the direct regulation of COX19 mRNA by the pathway. Alternatively, COX23 mRNA contains a long 3'-UTR, but is indirectly regulated by the pathway under two conditions tested here. Analysis of the functionality of the NMD targeting features in COX23 mRNA showed that the COX23 3'-UTR is sufficient to trigger NMD. The regulation of mRNAs involved in mitochondrial copper metabolism by NMD is physiologically significant because excess copper enhances growth of NMD mutants on a non-fermentable carbon source. These findings suggest that regulation of mRNAs encoding homologous proteins by NMD can be differential depending on environmental copper levels. Furthermore, these findings suggest copper ion homeostatic mechanisms in the mitochondria occur at the mRNA level via the NMD pathway.
\end{abstract}

Keywords Saccharomyces cerevisiae $\cdot$ mRNA $\cdot$ mRNA decay $\cdot$ Mitochondrial copper homeostasis $\cdot$ Nonsense-mediated mRNA decay

\section{Introduction}

The nonsense-mediated mRNA decay (NMD) pathway is a highly conserved mRNA degradation pathway found in all tested eukaryotes from yeast to humans. In Saccharomyces cerevisiae, $\sim 5-10 \%$ of the transcriptome is affected when NMD is non-functional (He et al. 2003; Guan et al. 2006; Celik et al. 2017). Similar results were observed in studies involving Drosophila melanogaster and humans (Mendell et al. 2004; Rehwinkel et al. 2005; Wittmann et al. 2006).

Communicated by M. Kupiec.

Bessie W. Kebaara

Bessie_Kebaara@Baylor.edu

1 Department of Biology, Baylor University, One Bear Place \#97388, Waco, TX 76798, USA

2 University of Wisconsin-Madison, Madison, WI 53706, USA
Upf1p, Upf2p and Upf3p are three core NMD factors that are required for a functional NMD pathway in all organisms.

NMD was first identified as a pathway that degrades premature termination codon (PTC) containing mRNAs, therefore preventing the synthesis of potentially harmful truncated proteins. NMD is now also recognized as a pathway that degrades natural mRNAs that encode fully functional proteins. Thus, NMD plays dual roles, one in mRNA surveillance and a second in regulation of gene expression. NMD-mediated degradation of natural mRNAs has been observed in diverse organisms including $S$. cerevisiae, $D$. melanogaster, Caenorhabditis elegans, plants and humans.

Natural mRNAs regulated by NMD are either direct or indirect targets. Direct NMD targets have significantly altered mRNA decay rates in cells with a functional versus non-functional NMD pathway. Conversely, indirect NMD targets have comparable decay rates in cells with a functional and non-functional NMD pathway. mRNAs that are direct 
targets typically have a variety of recognized NMD-inducing features in eukaryotes. In S. cerevisiae, these features include translated upstream open reading frames (uORF) (He et al. 2003; Gaba et al. 2005; Guan et al. 2006; Johansson and Jacobson 2010), out of frame initiation of translation (also known as "leaky scanning") (Welch and Jacobson 1999; Guan et al. 2006), inefficient and alternatively spliced pre-mRNAs (He et al. 1993; Guan et al. 2006), ribosomal frameshift signals (Belew et al. 2011; Celik et al. 2017), and atypically long 3'-untranslated regions (UTR) (Guisbert et al. 2007; Kebaara and Atkin 2009; Deliz-Aguirre et al. 2011). In addition, regulation of natural mRNAs by NMD can be growth condition specific. These observations have led to the hypothesis that these NMD targeting features can function in specific cellular context and environmental conditions (Peccarelli et al. 2016).

Our previous studies found that $S$. cerevisiae cells with an inactive NMD pathway are more tolerant of toxic copper levels (Deliz-Aguirre et al. 2011). To examine this phenotype further, we investigated a subset of mRNAs involved in copper homeostasis to gain insight into their regulation by NMD. Understanding the role NMD plays in copper homeostasis is important because copper is essential for cellular function but can be toxic in excess. Additionally, understanding copper homeostatic mechanisms in yeast will provide insight into the regulatory mechanisms used in other organisms as these homeostatic mechanisms have been conserved throughout evolution (De Freitas et al. 2003).

Analysis of a set of mRNAs involved in copper homeostasis revealed that a subset of these mRNAs function in mitochondrial copper utilization. Copper is an essential metal in the mitochondria for the functions of cytochrome $c$ oxidase $(\mathrm{CcO})$, and copper, zinc-superoxide dismutase (Sod1). The mRNAs we examined here are involved with $\mathrm{CcO}$ metallation. Cox 17p was initially identified as an $S$. cerevisiae $\mathrm{CcO}$ assembly mutant that was unable to carry out respiratory growth in the presence of $\mathrm{CcO}$ components. Subsequently, Cox 17p was found to be a copper-binding protein that delivers copper to $\mathrm{CcO}$ through two copper-binding intermembrane space-associated proteins Sco1 and Cox11. Mice lacking $\mathrm{COX} 17$ function show impaired $\mathrm{CcO}$ activity and die early in utero. Cox $19 p$ and Cox $23 p$ are homologs of Cox 17p and are required for the assembly of $\mathrm{CcO}$ and for respiration. Cox 17p, Cox 19p and Cox $23 p$ contain twin cysteine- $\mathrm{x}_{9}$-cysteine (twin $\mathrm{Cx}_{9} \mathrm{C}$ ) motifs and all have human orthologues (Longen et al. 2009). Cox23p is a mitochondrial intermembrane space protein that functions in mitochondrial copper homoeostasis. Cox 19p is found in the cytosol and the mitochondrial intermembrane space and is required for the assembly of $\mathrm{CcO}$.

Since COX17, COX19 and COX23 mRNAs encode functionally related proteins, we examined the extent to which COX17 mRNA is an NMD target based on our earlier observation that $C O X 19$ and $C O X 23$ mRNAs are NMD targets in rich media. We previously found that COX19 and COX23 mRNAs have atypically long 3'-UTRs, but are differentially regulated by the pathway in rich media. The COX19 mRNAs long 3'-UTR contributes to the degradation of the mRNA by NMD. Alternatively, despite having a long 3'-UTR, COX23 mRNA is indirectly regulated by the pathway in rich media (Peccarelli et al. 2016). Since these mRNAs have NMD targeting features, the pathway could directly regulate them in select environmental conditions. For example, we previously found that $M A C 1 \mathrm{mRNA}$ was directly regulated by NMD in rich media, but not regulated under low copper conditions, when the Mac1p is required (Peccarelli et al. 2016). Thus, we examined whether environmental copper levels affect the NMD-mediated regulation of these three mRNAs. Additionally, we examined the functionality of the NMD-targeting features in COX23 mRNA. We investigated the extent to which the long 3'-UTR is sufficient to target $C O X 23 \mathrm{mRNA}$ to NMD by replacing it with the short CYC1 3'-UTR which known to not trigger NMD. This enabled us to understand if the COX23 mRNA 3'-UTR was too short to activate NMD, is present in an incorrect context in $C O X 23$, or whether the NMD targeting features in COX23 function only under specific environmental conditions not examined here.

NMD mutants have respiratory impairments when grown on non-fermentable carbon sources. This growth impairment could be due to the accumulation of aberrant products interfering with respiratory function or altered expression of mRNAs and consequently proteins involved in mitochondrial copper homeostasis. Interestingly, yeast strains lacking COX17, COX19 and COX23 show impaired growth on non-fermentable carbon sources (Longen et al. 2009). In addition, this phenotype is observed when mitochondrial copper homeostasis is misregulated. We examined the extent to which the respiratory defect observed in NMD mutants is due to an imbalance in copper homeostatic mechanisms in the mitochondria. NMD mutants grown on lactate, a nonfermentable carbon source, under normal copper conditions, have impaired growth relative to wild-type cells. Interestingly, growth under the same conditions with media supplemented with excess copper recovered the growth defect of the NMD mutants. Additionally, we found that overexpression of COX19 results in increased tolerance of wild-type cells to elevated copper levels.

\section{Materials and methods}

\section{Yeast strains}

All S. cerevisiae strains and genotypes used in this study are listed in Table 1. 
Table 1 Saccharomyces cerevisiae strains used in this study

\begin{tabular}{|c|c|c|}
\hline Yeast strain & Genotype & Source \\
\hline W303 & a, ade2-1, ura3-1, his3-11,15, trp1-1, leu2-3,112, can1-101 & Wente et al. (1992) \\
\hline AAY320 & a, ade2-1, ura3-1, his3-11,15, trp1-1, leu2-3,112, can1-100, UPF1::URA3 (upf- $\Delta 2)$ & Kebaara et al. (2003) \\
\hline AAY327 & a, ade2-1, ura3-1, his3-11,15, trp1-1, leu2-3,112, can1-100, UPF1::TRP (upf1- $\Delta 6$ ) & Kebaara et al. (2003) \\
\hline AAY334 & a, ADE2, ura3-1 or ura3-52, his3-52, his3-11,15, trp1-1, leu2-3,112, rpb1-1 & Kebaara et al. (2003) \\
\hline AAY335 & a, ADE2, ura3-1 or ura3-52, his3-52, his3-11,15, trp1-1, leu2-3,112, rpb1-1, upf1-42 (URA3) & Kebaara et al. (2003) \\
\hline HFY1300 & MAT $\alpha$ ade2-1 ura3-1 his3-11,15 trp1-1 leu2-3,112 trp1-1 can1-100 UPF1 nmd2::HIS3 UPF3 & He and Jacobson (1995) \\
\hline HFY861 & MATa ade2-1 ura3-1 his3-11,15 trp1-1 leu2-3,112 trp1-1can1-100 UPF1 NMD2 upf3::HIS3 & He et al. (1997) \\
\hline
\end{tabular}

\section{Growth of yeast strains}

Yeast strains were maintained and grown using standard techniques (Ausubel et al. 1998). For analysis of COX17, COX19 and COX23 mRNAs under low copper conditions, wild-type and NMD mutant yeast cells were grown in low copper complete minimal (CM) media. This media contained yeast nitrogen base without copper ( $\mathrm{YNB}-\mathrm{CuSO}_{4}-\mathrm{FeCl}_{3}$ ) and $100 \mu \mathrm{M}$ bathocuproinedisulfonic acid (Sigma-Aldrich). Glassware used in these experiments was soaked in $10 \%$ nitric acid overnight to remove trace amounts of copper. All yeast cells used for low-copper northerns were initially grown to saturation in CM media and then subcultured into copper-deficient media in acid-washed glassware.

To analyze COX17, COX19 and COX23 mRNAs under high copper conditions, wild-type and NMD mutant yeast cells were grown in CM media supplemented with $100 \mu \mathrm{M}$ copper (high copper media). As with the low copper conditions, the yeast cells were first grown to saturation in complete minimal media then subcultured into media supplemented with $100 \mu \mathrm{M}$ copper.

\section{RNA methods}

For all mRNA steady-states and half-life experiments total $S$. cerevisiae RNA was used. Yeast cells cultured in the different conditions described above were harvested at midlog phase as described in Peccarelli and Kebaara (2014). Total RNA was extracted from harvested cells using the hot phenol method. $15 \mu \mathrm{g}$ of total RNA was resolved on $1.0 \%$ agarose-formaldehyde gels for all steady-state and half-life northerns. Then, the RNA was transferred to GeneScreen Plus ${ }^{\circledR}$ (PerkinElmer, Boston, MA, USA) nylon membranes using the NorthernMax ${ }^{\mathrm{TM}}$ Complete Northern Blotting kit (Thermo Fisher Scientific, Carlsbad, CA, USA) transfer protocol. Northern blots were probed with oligolabeled DNA probes that were labeled with $\left[\alpha-{ }^{32} \mathrm{P}\right]$ dCTP using the RadPrime DNA Labeling System (Thermo Fisher Scientific, Carlsbad, CA, USA). All DNA probes were generated by PCR. Northern blots were phosphorImaged ${ }^{\mathrm{TM}}$ using a
Typhoon Phosphorimager (Amersham Pharmacia Biotech, Inc.).

For low copper controls, all northerns with RNA extracted from yeast cells grown under low copper conditions were probed with CTR1. CTRI encodes a high affinity copper transporter of the plasma membrane. Diminished copper levels result in increased CTRI expression. For high copper controls, all northerns with RNA extracted from yeast cells grown in $100 \mu \mathrm{M}$ copper were probed with CUP1. CUP1 encodes a metallothionein that binds copper. The $C U P 1$ gene is induced by the Ace 1 transcription factor when yeast cells are exposed to elevated copper levels. Increases in copper levels result in increased CUPI expression. For NMD controls, all northerns were probed with CYH2 pre-mRNA to confirm the NMD phenotype of the yeast strains. $\mathrm{CYH} 2$ pre-mRNA is a known NMD target, while $C Y H 2$ mRNA is not (He et al. 1993). We used SCRI RNA as a loading control to normalize RNA levels. SCRI is an RNA polymerase III transcript that is not sensitive to NMD. $C Y H 2$ pre-mRNA, $C Y H 2$ mRNA and $S C R 1$ RNA are not known to be responsive to environmental copper levels. All northerns were quantified using ImageQuant software. Sigmaplot, Version 13 software was used to calculate halflives as described in Peccarelli and Kebaara (2014).

\section{3'RACE}

$3^{\prime} \mathrm{RACE}$ was used to determine the length of the $3^{\prime}$-UTRs as described in Kebaara et al. (2012) using the 3'RACE System for Rapid Amplification of cDNA Ends kit (Thermo Fisher Scientific, Carlsbad, CA, USA). Yeast total RNA used for steady-state northern blots was used to generate cDNA using SuperScript ${ }^{\mathrm{TM}}$ II RT (Thermo Fisher Scientific, Carlsbad, CA, USA). Subsequently, the cDNA was used as the template for all primary PCR reactions. Primary PCR reactions used the Abridged Universal Amplification Primer (AUAP) from the 3'RACE kit in combination with gene-specific primers. The primary PCR product served as a template for the nested PCR reactions. All nested PCR reactions utilized gene specific primers. PCR products for both primary and nested reactions were run on $1.5 \%$ agarose gels. 


\section{DNA methods}

To create a fusion construct for COX23 mRNA, the long 3'-UTR from $C O X 23$ was amplified by PCR. Subsequently, the 5'-UTR and ORF of a second gene, $C Y C 1$, was amplified by PCR. CYCl mRNA is not an NMD target. Third, ligation-mediated PCR fused the two PCR fragments. To generate $C Y C 1 C O X 233^{\prime}$-UTR, the $C Y C 15^{\prime}$-UTR and ORF were fused to $350 \mathrm{nt}$ from the COX23 3'-UTR. The fusion construct was then inserted into the TOPO-TA cloning vector according to manufacturer's instructions and sent for sequencing to confirm that the correct fusion construct was created. Next, CYC1COX23 3'-UTR was digested with BamHI and SacI before ligation into pRS425 digested with the same enzymes.

\section{Results}

\section{COX17 and COX19 mRNAs are direct NMD targets in rich media, while COX23 mRNA is an indirect target}

Because the proteins encoded by COX17, COX19 and COX23 mRNAs are homologues and function in mitochondrial copper utilization, we examined whether they are regulated by NMD similarly. In agreement with our previous study (Peccarelli et al. 2016), we found that in rich media the major COX19 mRNA isoform is directly regulated by NMD, while in the same conditions COX23 is indirectly regulated by the pathway (Fig. $1 \mathrm{~b}, \mathrm{c}$; Table 2). Measurement of COX17 mRNA half-life in wild-type and NMD mutant strains showed that the COX17 mRNA is a direct NMD target in rich media (Fig. 1a). In these conditions, the half-life of COX17 mRNA in the wild-type strain (UPF1) was $6.3 \mathrm{~min}$ relative to $17.0 \mathrm{~min}$ in the NMD mutant strain.

Since Cox 17p, Cox 19p and Cox 23p function in $\mathrm{CcO}$ metallation and are regulated differentially by NMD in rich media, we examined the extent to which environmental conditions affect NMD-mediated regulation of these mRNAs. To test this, we determined the steady-state and half-lives of the three mRNAs under copper deplete and excess copper conditions.

\section{Differential regulation of COX17, COX19 and COX23 mRNAs by NMD under low copper conditions}

To investigate the effect of environmental copper levels on regulation of COX17, COX19 and COX23 mRNAs by NMD, we measured the steady-state and half-lives of the mRNAs in wild-type and NMD mutant yeast strains grown under low copper conditions [media containing bathocuproinedisulfonic acid (BCS)]. To confirm that low copper conditions were achieved the northern blots were first probed with CTR1. CTRI encodes a high-affinity copper transporter of the plasma membrane. Reduced copper levels result in increased CTRI expression. Under low copper conditions, CTR1 mRNA levels were elevated in both wild-type and NMD mutants (Fig. 2a). Additionally, under these conditions the CTR1 mRNA is not an NMD target. The half-life of CTR $1 \mathrm{mRNA}$ in the wild-type strain was $5.0 \mathrm{~min}$ relative to $5.7 \mathrm{~min}$ in the NMD mutant strain (Fig. 2a, bottom panel). Furthermore, under these conditions NMD is functional as shown by the steady-state accumulation and stabilization of the $C Y H 2$ pre-mRNA in the NMD mutants (Fig. 2b).

Under low copper conditions, one COX17 mRNA isoform was detected on northern blots and the mRNA was not regulated by NMD. COX17 mRNA did not accumulate to higher levels in NMD mutants and was not stabilized in cells with a non-functional NMD pathway (Fig. 3a; Table 2). In these conditions, the half-life of COX17 mRNA in the wildtype strain $(U P F 1)$ was $6.7 \mathrm{~min}$ relative to $7.5 \mathrm{~min}$ in the NMD mutant (Fig. 3a; Table 2). In the wild-type strain, the half-life was similar to the observed half-life in rich media. While in the NMD mutant, the COX17 mRNA half-life was 2.5-fold longer in rich media compared with low copper conditions (Figs. 1a, 3a).

Conversely, COX19 mRNA accumulated to higher levels in NMD mutants and was stabilized in yeast cells with a non-functional NMD pathway (Fig. 3b; Table 2). COX19 mRNA accumulated $3.5( \pm 0.8)$-fold higher in NMD mutants relative to the wild-type strain. Under these conditions, the major COX19 mRNA isoform is degraded faster in wildtype cells relative to the NMD mutant (Fig. 3b; Table 2). The half-lives were similar in wild-type strains grown under low copper and rich media (Figs. 1b, 2b). On the other hand, COX19 mRNA half-life is 3.2-fold longer in NMD mutants grown in rich media relative to low copper conditions (Figs. 1b, 3b).

COX23 mRNA did not accumulate to higher levels in NMD mutants and was not stabilized in yeast cells with a non-functional NMD pathway (Fig. 2c; Table 2). COX23 mRNA accumulated $0.9( \pm 0.4)$-fold higher in NMD mutants relative to wild-type cells. Under these conditions, COX23 mRNA is degraded at comparable rates in wild-type cells relative to NMD mutants (Fig. 3c; Table 2). The half-life of COX23 mRNA in the wild-type strain (UPF1) was $7.7 \mathrm{~min}$ relative to $7 \mathrm{~min}$ in the NMD mutant strain. The half-life of COX23 mRNA was shorter in both wild-type and NMD mutant strains grown under low copper conditions relative to yeast strains grown in rich media (Figs. 1c, 3c).

Overall, under low copper conditions COX19 mRNA accumulate to higher levels in the NMD mutants relative to the wild-type strains, while COX17 and COX23 mRNAs do not accumulate to higher levels (Fig. 3a-c). Interestingly, under these conditions, COX19 mRNA is the only NMD 


\section{A COX17 mRNA in YAPD}
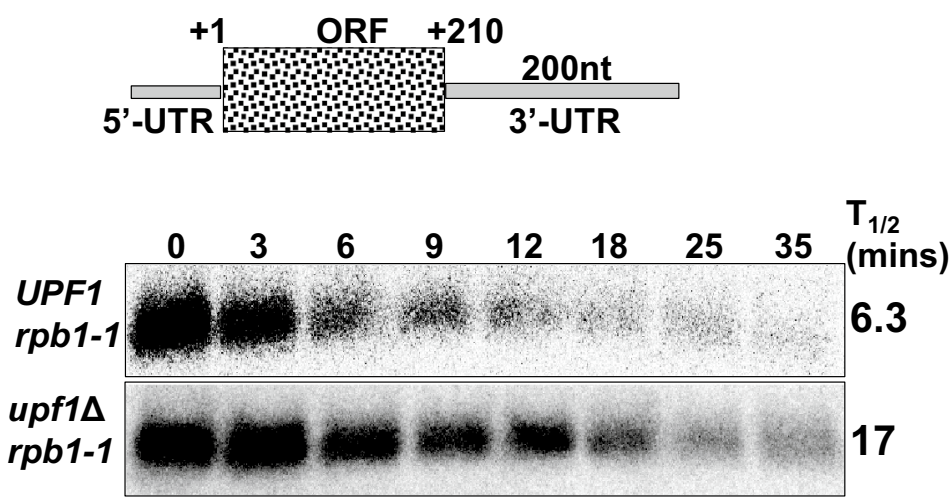

B COX19 mRNA in YAPD
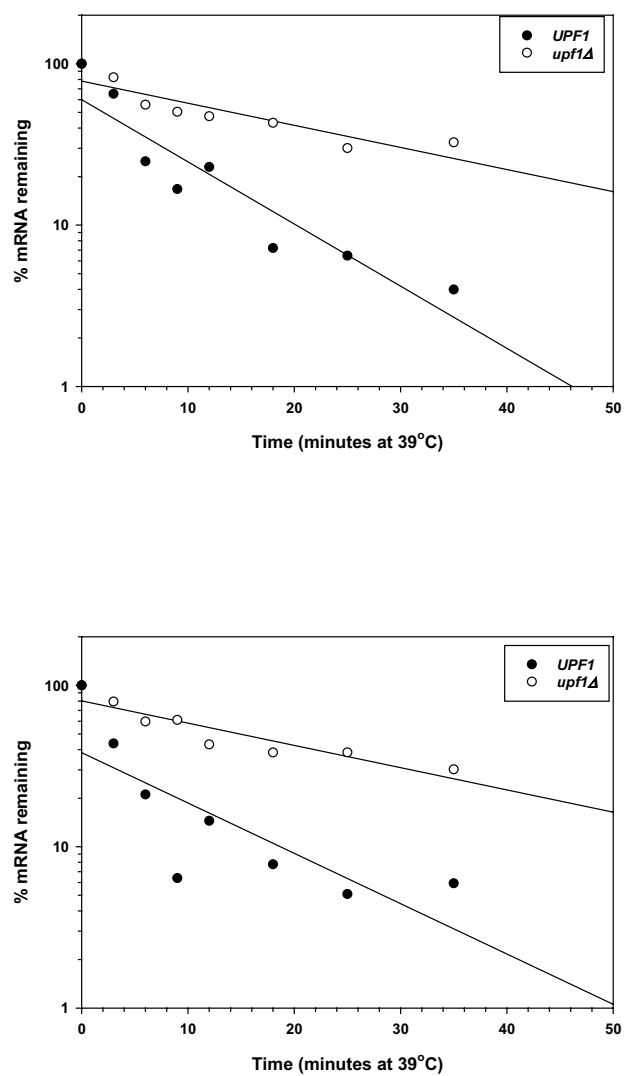

5.9

47.5

$T_{1 / 2}$

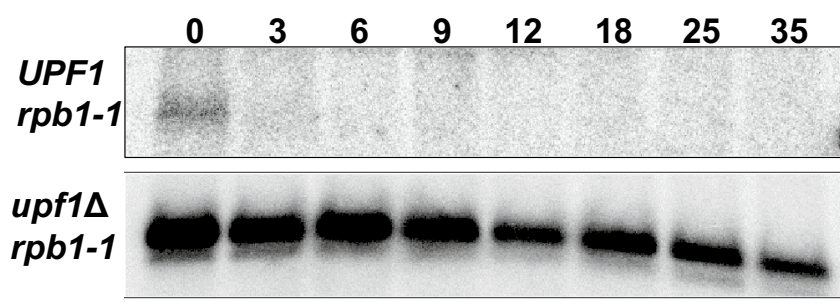

(mins)

\section{COX23 mRNA in YAPD}
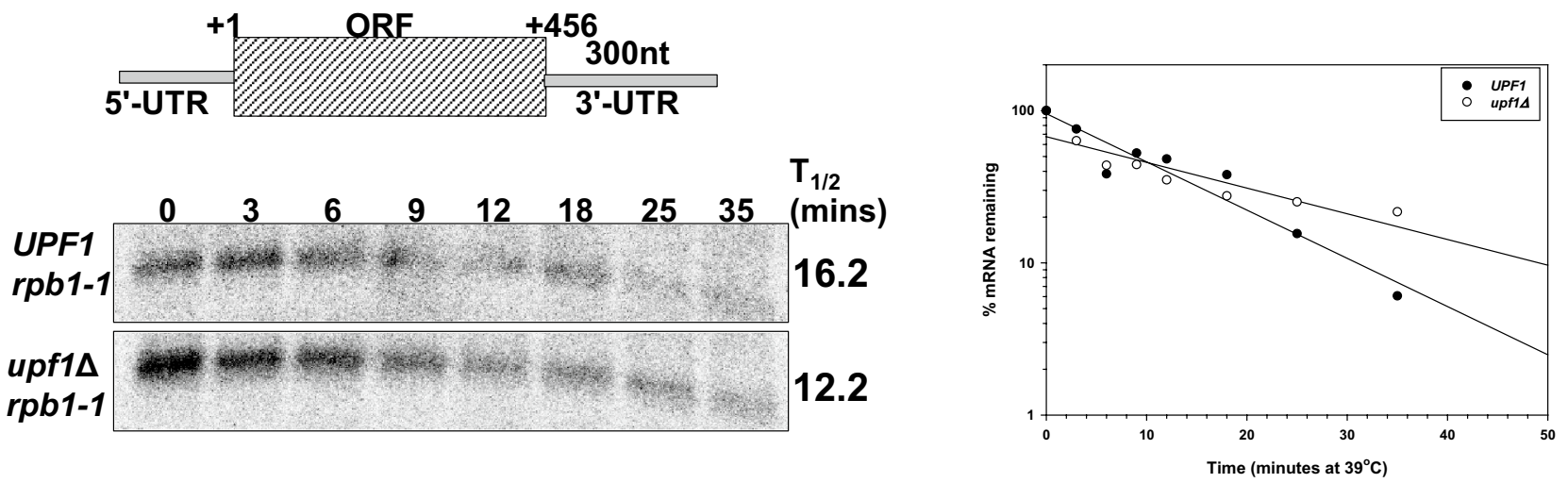

Fig. 1 COX17 and COX19 mRNAs are direct NMD targets in rich media while $C O X 23$ mRNA is an indirect target. Schematic representations of COX17, COX19 and COX23 mRNAs (a-c, respectively). Representative half-life northern blots were measured with total RNA extracted from wild-type strain AAY334 (UPF1 rpb1-1; Kebaara et al. 2003) and NMD mutant strain AAY335 (upf1s rpb1-1; Kebaara et al. 2003). Yeast cells were harvested over a 35-min period at different time points indicated above the northern blots. The northern blots were probed with radiolabeled DNA from the COX23, COX19 and COX17 ORFs, respectively. For controls, the membranes were probed with $\mathrm{CYH} 2$ and SCR1 DNA. The half-lives were determined using SigmaPlot graphs and are shown to the right of each northern blot. Typical SigmaPlot graphs showing the decay rate of COX17 (a), COX19 (b) and COX23 (c) mRNAs in UPF1 and upf1A yeast strains are shown to the right of the half-life northern blots. The half-life measurements are an average of at least three independent experiments and are calculated based on the time required for half of the original RNA to degrade 
Table 2 COX17, COX19 and COX23 mRNA half-lives were measured in isogenic wild-type (UPF1 rpb1-1) and NMD mutants (upf1D rpb1-1)

\begin{tabular}{llccc}
\hline Growth media & Yeast strain & COX17 mRNA & COX19 mRNA & COX23 mRNA \\
\hline YAPD & UPF1 & $6.3 \pm 1.5$ & $5.94 \pm 1.44^{\mathrm{a}}$ & $16.19 \pm 2.43^{\mathrm{a}}$ \\
YAPD & upf1 & $17 \pm 4$ & $47.48 \pm 22.51^{\mathrm{a}}$ & $12.20 \pm 2.05^{\mathrm{a}}$ \\
Low $\mathrm{Cu}$ & $U P F 1$ & $6.7 \pm 0.6$ & $3.7 \pm 0.6$ & $7.7 \pm 4.0$ \\
Low $\mathrm{Cu}$ & upfl & $7.5 \pm 1.0$ & $14.7 \pm 4.2$ & $7 \pm 2.6$ \\
$100 \mu \mathrm{M} \mathrm{Cu}$ & $U P F 1$ & $12.3 \pm 4.7$ & $4.0 \pm 1.0$ & $10.7 \pm 4.5$ \\
$100 \mu \mathrm{M} \mathrm{Cu}$ & upfl & $13 \pm 3.0$ & $12.3 \pm 0.57$ & $9.6 \pm 4.7$ \\
\hline
\end{tabular}

All yeast strains used were grown under the conditions indicated in the table

$N D$ not determined

${ }^{\text {a}}$ Half-lives were reported previously (Peccarelli et al. 2016)

A CTR1 mRNA in BCS (low Cu)

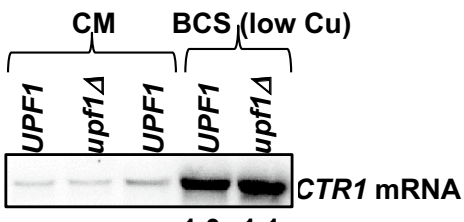

1.01 .1
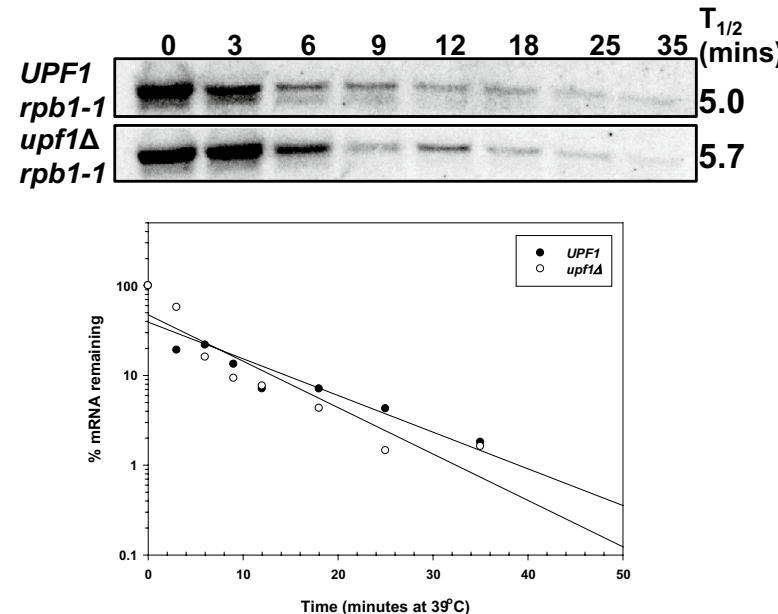

Fig. 2 The NMD pathway is functional under low copper conditions and the CTR1 mRNA is not regulated by NMD under these conditions. Representative steady-state mRNA accumulation levels were measured with RNA extracted from isogenic wild-type strain W303 (UPF1) and NMD mutant AAY320 (upflA) yeast strains grown in complete minimal media containing bathocuproinedisulfonic acid (BCS). Half-life northern blots with total RNA extracted from wildtype strain AAY334 (UPF1 rpb1-1; Kebaara et al. 2003) and NMD mutant strain AAY335 (upfls rpb1-1; Kebaara et al. 2003). Yeast cells were harvested over a 35 -min time period at different time
B CYH2 mRNA in BCS (low Cu)
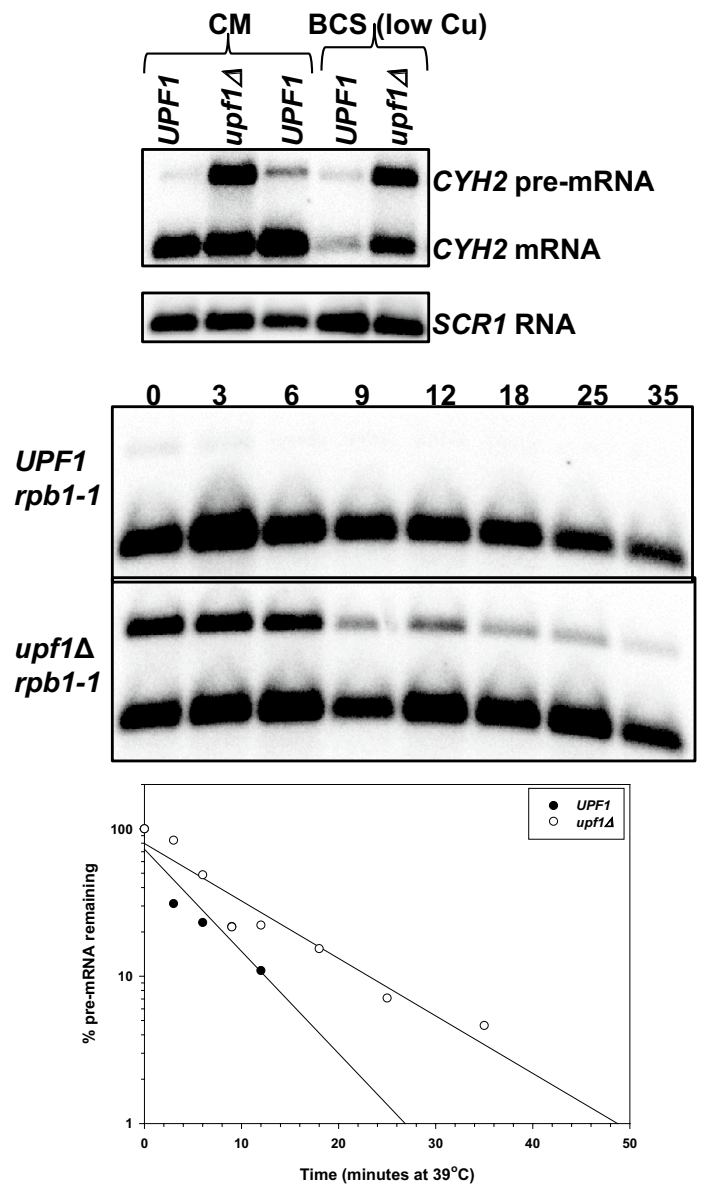

points indicated above the northern blots. The northern blots were sequentially probed with radiolabeled DNA from the CTR1 (a), CYH2 and SCR1 (b). CTR1 encodes a high-affinity copper transporter of the plasma membrane. Reduced copper levels result in increased CTRI mRNA expression. Typical SigmaPlot graphs showing the decay rate of CTR1 (a) and CYH2 pre-mRNA in UPF1 and upflA yeast strains are shown below the half-life northern blots. The halflife measurements are an average of at least three independent experiments 
target, while COX23 and COX17 mRNAs escape degradation by the pathway (Fig. 3a-c, right panels; Table 2). This differs from what we observed with rich media where COX19 and COX17 mRNAs are direct NMD targets.

\section{Under media supplemented with $100 \mu \mathrm{M}$ copper COX17 mRNA is immune to NMD-mediated degradation, COX23 mRNA is indirectly regulated, while COX19 mRNA is directly regulated by the pathway}

To further investigate the extent to which regulation of COX17, COX19 and COX23 mRNAs by NMD is dependent on environmental copper levels, we examined the regulation of the mRNAs in wild-type and NMD mutant yeast strains grown in excess copper (media containing $100 \mu \mathrm{M}$ copper). The elevated copper conditions were verified by probing the northern blots with CUP1 mRNA. CUP1 encodes a metallothionein that binds copper. The $C U P 1$ gene is induced by the Ace1 transcription factor when yeast cells are exposed to elevated copper levels. Increases in copper levels result in increased $C U P 1$ expression. CUP 1 mRNA accumulated $4.25( \pm 0.5)$-fold higher in wild-type yeast strains grown under high copper conditions relative to yeast strains grown in regular copper levels. Additionally, CUP1 mRNA accumulated 14.33 ( \pm 1.4$)$-fold higher in NMD mutant strains grown under elevated copper levels (Fig. 4a, left panel). Comparing CUP1 mRNA levels in wild-type and NMD mutants shows that the mRNA does not accumulate in the NMD mutant under normal or elevated copper conditions (Fig. 4a, left panel). Thus, CUP1 mRNA is not an NMD target in rich media or under elevated copper conditions. CUP1 mRNA half-life could not be determined within the time points utilized in these experiments, the mRNA did not decay within the 35-min time period in either wild-type or NMD mutant yeast strains. It appears that, under elevated copper levels CUP1 mRNA is very stable and not regulated by the NMD pathway (Fig. 4a, left panel).

Furthermore, the NMD pathway is functional under high copper conditions as validated by $\mathrm{CYH} 2$ pre-mRNA and mRNA steady-state accumulation and half-lives in excess copper conditions (Fig. 4b). We tested the NMD status of yeast cells grown under elevated copper levels because excess copper can induce stress (like hypoxia and ER stress). Stress has been shown to inhibit NMD.

Under elevated copper levels, one COX17 mRNA isoform was detected and the mRNA was not regulated by NMD. COX17 mRNA did not accumulate to higher levels in NMD mutants and was not stabilized in yeast cells with a non-functional NMD pathway (Fig. 5a; Table 2). The halflife of COX17 mRNA in the wild-type strain was $12.3 \mathrm{~min}$ relative to $13 \mathrm{~min}$ in the NMD mutant strain. In wild-type strains, COX17 mRNA half-life was longer under elevated copper conditions relative to both low copper conditions and rich media. In NMD mutants, COX17 mRNA half-life was comparable in rich media and elevated copper levels but faster under low copper conditions (Fig. 5a; Table 2). These COX17 mRNA half-life observations are similar to low copper but unlike what we observed in rich media (Figs. 1a, 3a).

In addition, one major COX19 mRNA isoform was detected in wild-type and NMD mutant strains grown in media supplemented with $100 \mu \mathrm{M}$ copper. The major COX19 mRNA isoform accumulated to higher levels in NMD mutants. Steady-state accumulation levels of COX19 mRNA were threefold higher in wild-type and NMD mutant strains grown in complete minimal media relative to complete minimal media supplemented with $100 \mu \mathrm{M}$ copper. Under these conditions, $C O X 19$ mRNA was degraded faster in wild-type cells relative to NMD mutants. The half-life of COX19 mRNA in the wild-type strain was 4.0 min relative to $12.3 \mathrm{~min}$ in the NMD mutant strain. This was comparable to what we observed with rich media and under low copper (Fig. 5b; Table 2).

Further, one COX23 mRNA isoform was detected in wild-type and NMD mutant strains grown on media containing $100 \mu \mathrm{M}$ copper. Steady-state accumulation levels of COX23 mRNA were comparable in wild-type (UPF1) and NMD mutant (upfls) strains grown in complete minimal or complete minimal supplemented with $100 \mu \mathrm{M}$ copper. Furthermore, under these conditions, the COX23 mRNA was an indirect NMD target (Fig. 5c; Table 2). The half-life of COX23 mRNA in the wild-type strain was 10.7 min relative to $9.7 \mathrm{~min}$ in the NMD mutant strain. These observations are comparable to rich media where we identified $\mathrm{COX} 23$ as an indirect NMD target (Table 2). The indirect regulation of COX23 mRNA by NMD under several environmental copper levels promoted us to examine the functionality of the NMD targeting feature within COX23 mRNA.

\section{The COX23 mRNA 3'-UTR is sufficient to target an NMD-insensitive transcript for NMD-mediated degradation}

Of the three mRNAs encoding proteins involved in metallation of $\mathrm{CcO}$ and regulated in an NMD-dependent manner, COX23 mRNA has an identifiable NMD targeting feature and is immune to degradation or indirectly regulated by the pathway in the conditions tested here. COX23 mRNA is an indirect NMD target in rich media and under elevated copper conditions (Figs. 1c, 5c) (Peccarelli et al. 2016). COX23 mRNA has an atypically long 3'-UTR of $300 \mathrm{nt}$ that does not appear to target the mRNA to NMD-mediated degradation. These observations are distinct from the other two mRNAs encoding functionally homologous proteins including $\mathrm{COX} 19$. COX19 mRNA has an atypically long 3'-UTR and was found to be directly regulated by NMD under all three conditions 


\section{A COX17 mRNA in BCS (low Cu)}
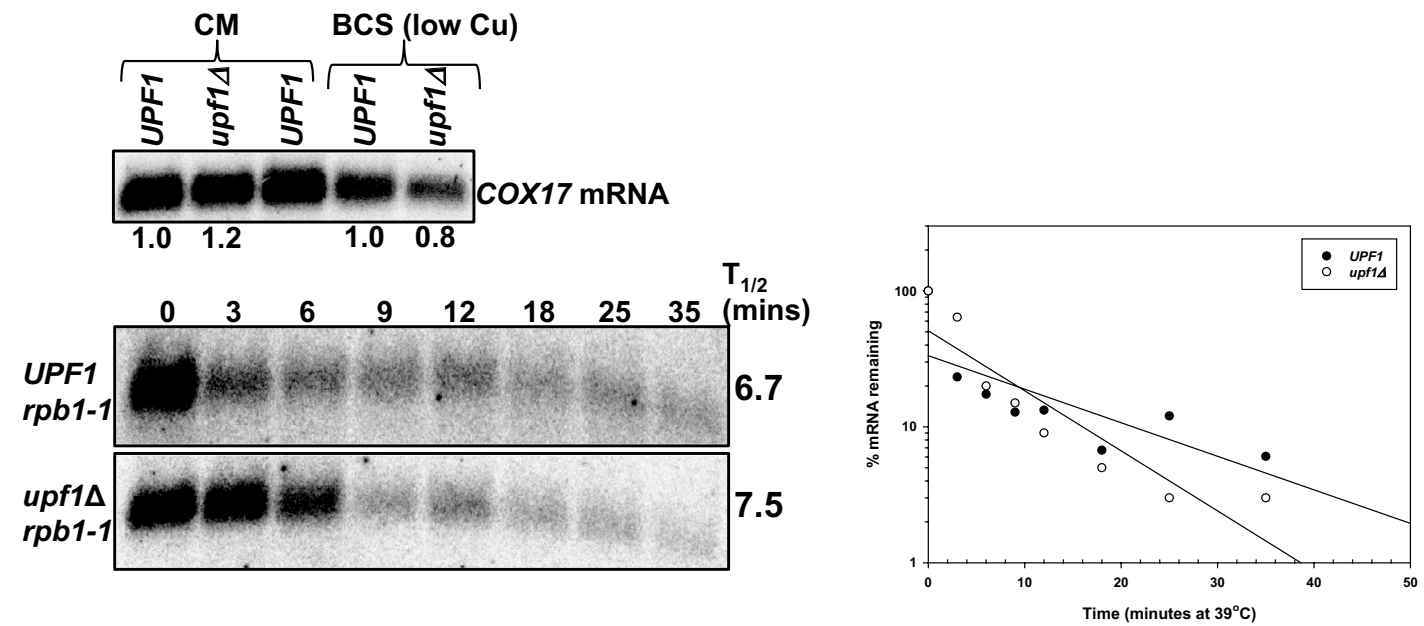

B COX19 mRNA in BCS (low Cu)
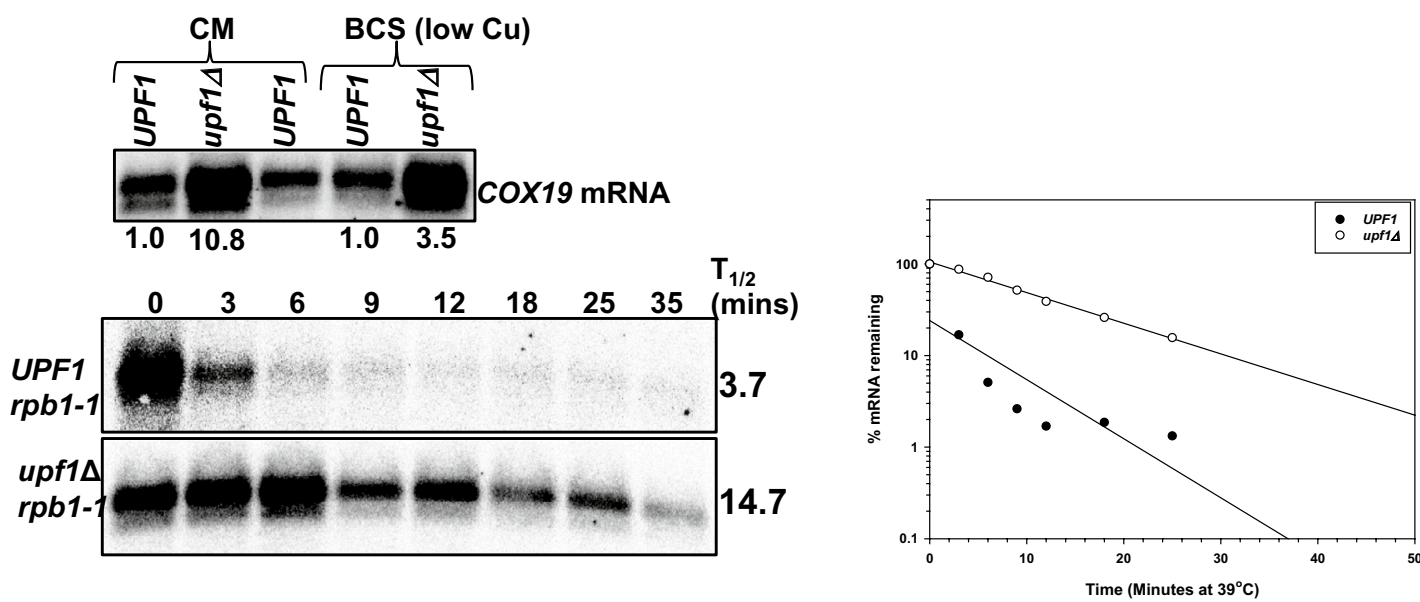

C COX23 mRNA BCS (low $\mathrm{Cu}$ )
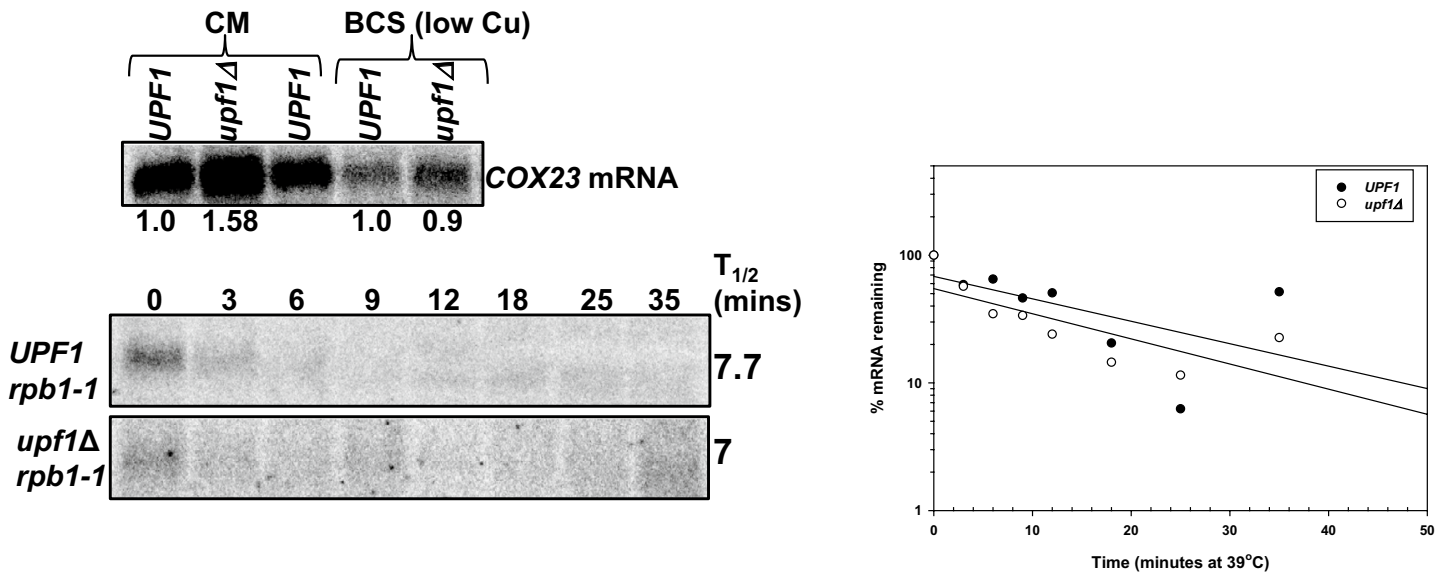

Fig. 3 COX23 mRNA is immune to NMD under low copper conditions; COX17 mRNA is indirectly regulated by the pathway, while COX19 mRNA is a direct NMD target under this conditions. Representative steady-state mRNA accumulation levels (a-c, top panels) were measured as described in Fig. 2. Half-life northern blots (a-c, bottom panels) with total RNA extracted from wild-type strain AAY334 (UPF1 rpb1-1; Kebaara et al. 2003) and NMD mutant strain AAY335 (upfls rpb1-1; Kebaara et al. 2003) were measured as described in Fig. 2. The northern blots were probed with radiolabeled DNA from the COX23, $C O X 19$, and $C O X 17$, respectively. The halflives were determined using SigmaPlot and are shown to the right of each northern blot. The half-life measurements are an average of at least three independent experiments 
tested here and previously (Figs. 1b, 3b, 5b) (Peccarelli et al. 2016). Furthermore, the 3 '-UTR of COX19 plays a role in the regulation of this mRNA by NMD (Peccarelli et al. 2014, 2016). It is possible that the COX 23 mRNA is an indirect NMD target in select conditions because the mRNAs 3'-UTR is in an incorrect context. Alternatively, this NMD targeting feature could target the COX23 mRNA to NMD in specific environmental conditions not tested here, as was previously observed with MACl mRNA (Peccarelli et al. 2016). To investigate if the COX23 3'-UTR is sufficient to target an NMDinsensitive mRNA to the pathway in defined media, we generated the CYCICOX23 3'-UTR fusion mRNA. The fusion

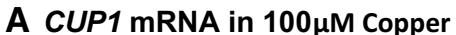
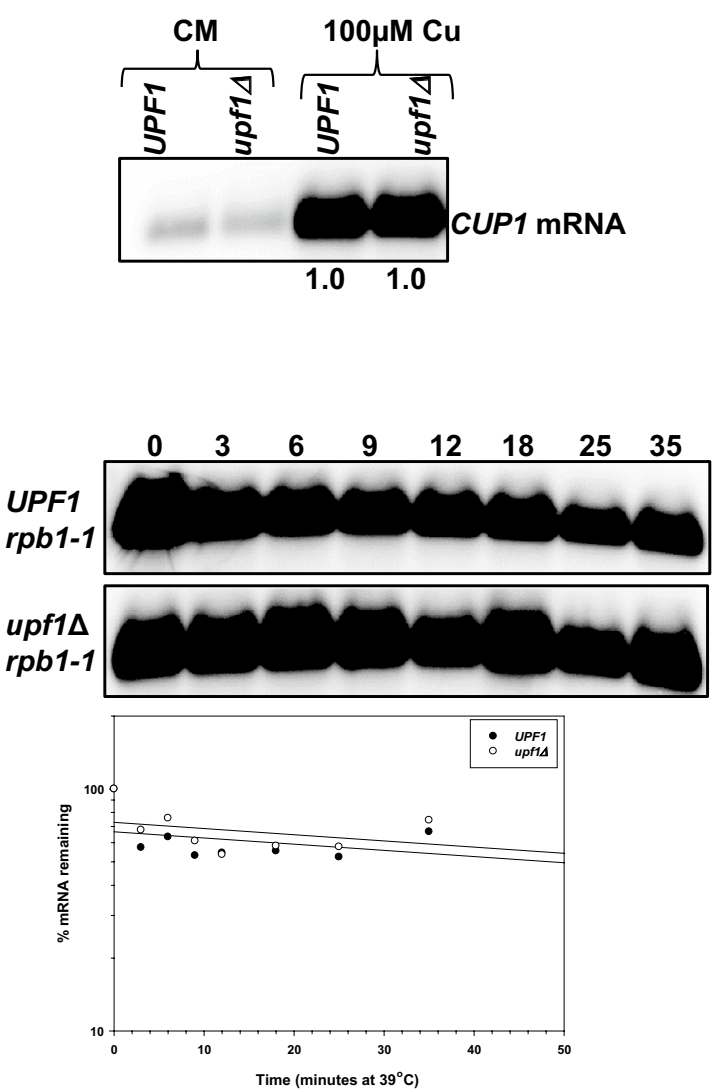

Fig. 4 The NMD pathway is functional under $100 \mu \mathrm{M}$ copper and the CUP1 mRNA is not an NMD target under this conditions. Steadystate mRNA accumulation levels were measured with RNA extracted from isogenic wild-type strain W303 (UPF1) and NMD mutant AAY320 (upfl $1 \Delta$ ) yeast strains grown in media containing $100 \mu \mathrm{M}$ copper (top panels). Half-life northern blots with total RNA extracted from wild-type strain AAY334 (UPF1 rpb1-1; Kebaara et al. 2003) and NMD mutant strain AAY335 (upfls rpb1-1; Kebaara et al. 2003) grown in media containing $100 \mu \mathrm{M}$ copper (bottom panels). Yeast cells were harvested as described in Fig. 2 and probed with radiolabeled DNA from the $C U P 1$ (a), $C Y H 2$ and SCRI (b). CUP1 encodes
mRNA contains the $5^{\prime}$-UTR and ORF from $\mathrm{CYCl}$ fused to the COX23 3'-UTR (Fig. 6a). The CYC1 mRNA, which encodes for iso-1-cytochrome $c$, was used because it has previously been utilized to study instability elements and is insensitive to the NMD pathway (Zaret and Sherman 1984; Peccarelli et al. 2014).

The CYC1COX23 3'-UTR mRNA was significantly less abundant than the endogenous $C Y C 1$ mRNA and accumulated $2.35( \pm 0.68)$-fold higher in the NMD mutants relative to the wild-type yeast strain (Fig. 6b). This observation suggests that the COX23 mRNA 3'-UTR contains a general mRNA destabilizing element. Additionally, the half-life of

\section{B CYH2 mRNA/ pre-mRNA in $100 \mu \mathrm{M} \mathrm{Cu}$}
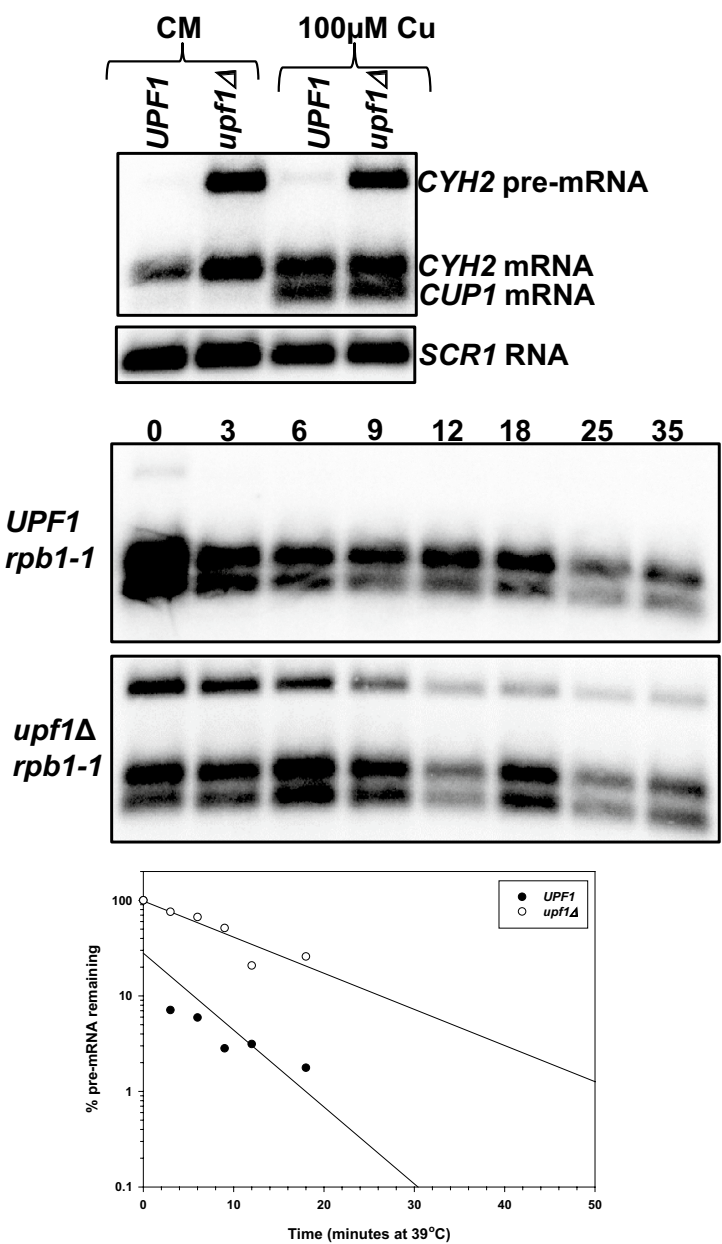

a metallothionein that binds copper. The bottom band on the $\mathrm{CYH} 2$ mRNA/pre-mRNA steady-state and half-live northern is the CUPI mRNA, which is highly overexpressed under high copper conditions. The CUP1 gene is induced by the Ace1 transcription factor when yeast cells are exposed to elevated copper levels. Increases in copper levels result in increased $C U P 1$ expression. Half-lives were determined using SigmaPlot, by measuring the time it takes for half of the original mRNA levels to degrade. The half-life graphs are shown below each half-life northern blot. The half-life measurements are an average of at least three independent experiments 


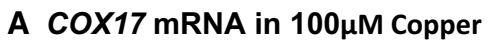
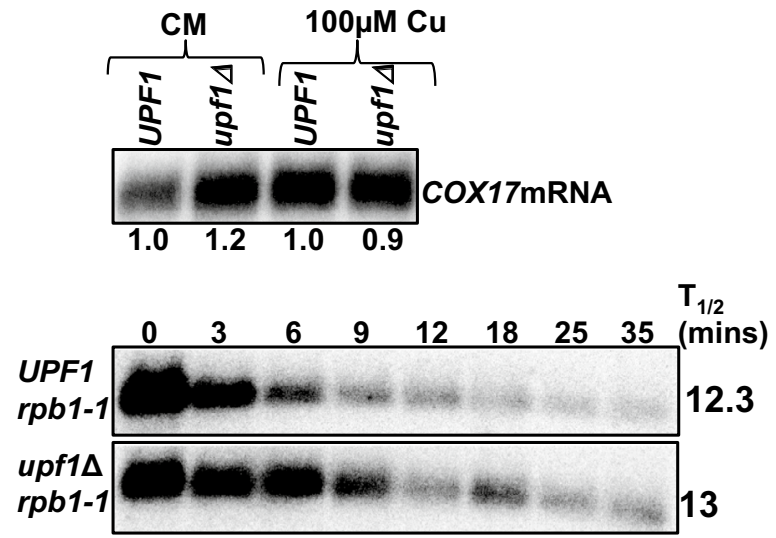

B COX19 mRNA in 100 $\mu \mathrm{M}$ Copper

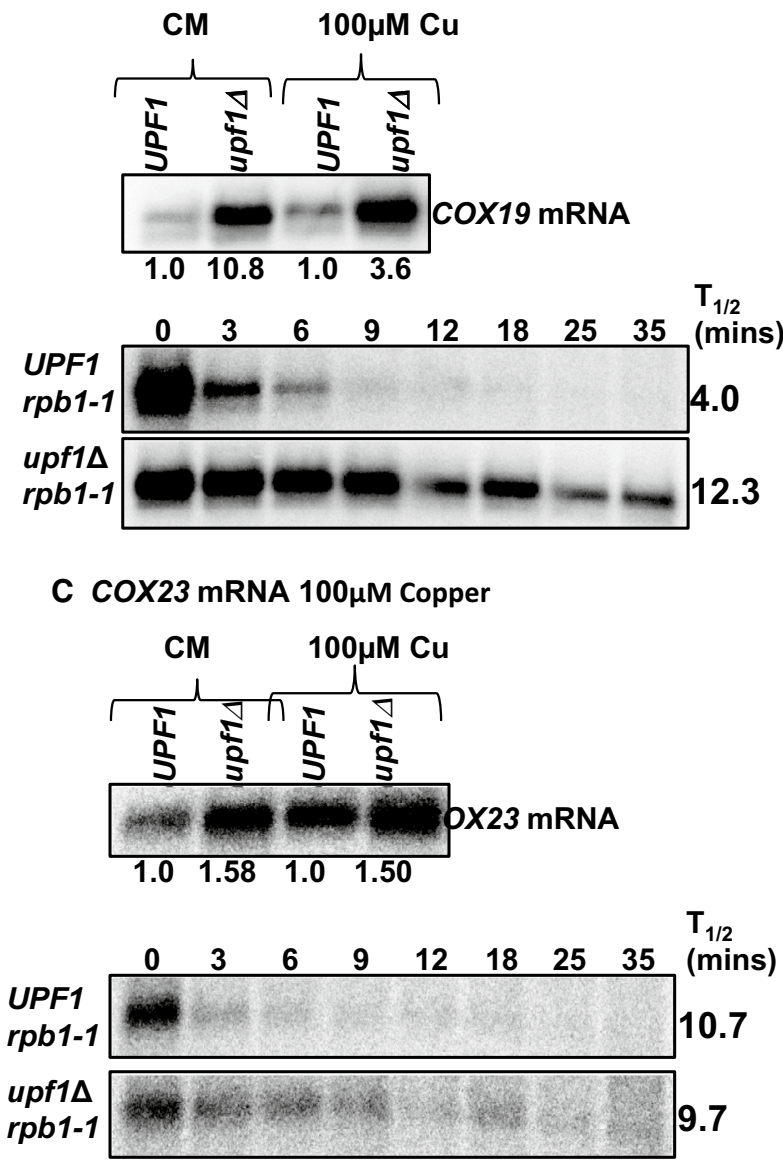

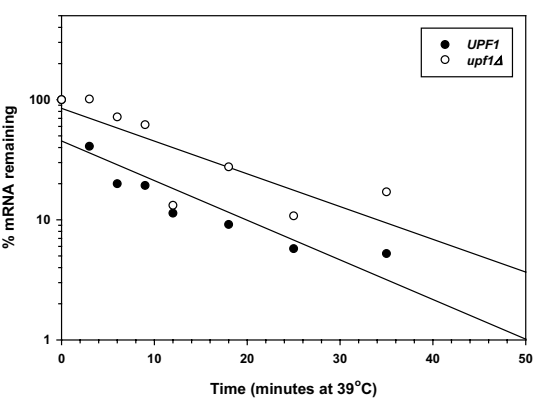
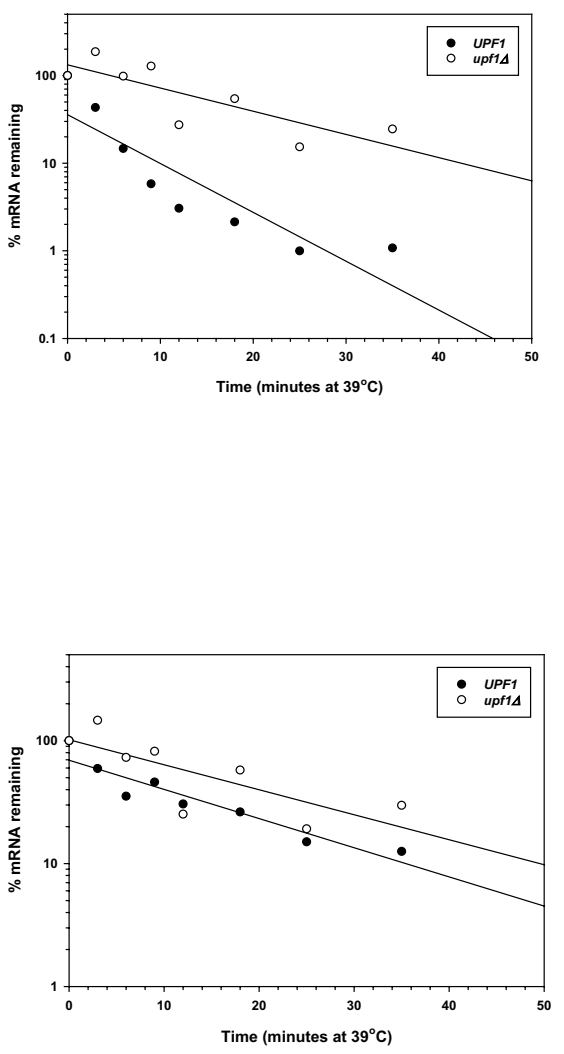

Fig. 5 COX19 mRNA is a direct NMD target under high copper conditions, while COX23 mRNA is an indirect target and COX17 mRNA is immune to degradation by the pathway. Representative steady-state mRNA accumulation levels (a-c, top panels) were measured with RNA as described in Fig. 4. Half-life northern blots (a-c, bottom panels) with total RNA extracted from wild-type strain AAY334 (UPF1 rpb1-1; Kebaara et al. 2003) and NMD mutant strain AAY335 (upf1s rpb1-1; Kebaara et al. 2003). Yeast cells were harvested as described in Fig. 4 and probed with radiolabeled DNA from the COX23, COX19, and COX17 ORFs, respectively. Half-lives were determined using SigmaPlot, by measuring the time it takes for half of the original mRNA levels to degrade. The half-life graphs are shown to the right of each half-life northern blot. The half-life measurements are an average of at least three independent experiments 
the CYC1COX23 3'-UTR mRNA in the wild-type strain was $14.20 \pm 5.50 \mathrm{~min}$ relative to $20.70 \pm 3.50 \mathrm{~min}$ in the NMD mutant strain (Fig. 6c). Although the values were somewhat close, the difference between the two half-lives is statistically significant, indicating that $\mathrm{CYClCOX} 233^{\prime}$-UTR fusion mRNA is directly regulated by NMD. These results suggest that the COX23 3'-UTR is sufficient to target an NMD-insensitive transcript to the pathway and supports the conclusion that the COX23 mRNA evades direct regulation by NMD in specific conditions.

\section{NMD mutants' respiratory impairments on non-fermentable carbon sources is recovered by elevated copper levels}

Previous studies have found that NMD mutants have respiratory impairments when grown on non-fermentable carbon sources. This growth defect could be due to accumulation of products interfering with respiratory function or altered expression of mRNAs involved in mitochondrial copper homeostasis. To test the extent to which NMD-mediated regulation of mRNAs involved in mitochondrial copper utilization affects this respiratory impairment, we grew wildtype and NMD mutant yeast strains on media containing lactate, a non-fermentable carbon source and supplemented the media with excess copper.

As we previously reported, NMD mutants are more tolerant of toxic copper levels when grown on glucose as a carbon source. This copper tolerance phenotype is clearly observed when wild-type and NMD mutants are grown on media containing $1 \mathrm{mM}$ copper. When wild-type, and NMD mutants were grown on media containing lactate as the carbon source, the NMD mutants had impaired growth (Fig. 7b, left panel). upf $1 \Delta$, upf $2 \Delta$, and $u p f 3 \Delta$ mutants all had equally reduced growth. Addition of excess copper to media containing lactate resulted in enhanced growth of all the strains predominantly the NMD mutants. Interestingly, upf $2 \Delta$ and upf $3 \Delta$ NMD mutant strains showed noticeably enhanced growth compared to the wild-type strain on media containing lactate with elevated copper (Fig. 7b, right panel). We also examined the effect overexpressing COX17, COX19 and COX23 has on wild-type and NMD mutants' strains grown on media containing elevated copper levels. Overexpression of $C O X 17$ and $C O X 23$ resulted in a phenotype similar to wild-type and NMD mutant yeast strains. The NMD mutant strain was more tolerant of elevated copper levels (Fig. 8a, c). Interestingly, overexpression of COX19 results in increased tolerance of the wild-type strain to elevated copper levels (Fig. 8b). Notably, COX19 mRNA is the only mRNA that was regulated by NMD in all the conditions tested here including elevated copper conditions. Thus, it appears that regulation of mRNAs involved in mitochondrial copper utilization by NMD affects growth of wild-type and NMD mutants on a non-fermentable carbon sources.

\section{Discussion}

The observation that in some conditions the NMD pathway differentially regulates mRNAs that encode structurally and functionally related proteins suggests that the regulation of these mRNAs maybe condition specific. This form of regulation would allow yeast cells to control the expression of specific mRNAs in response to environmental changes. Since these mRNAs encode proteins involved in mitochondrial copper utilization, copper levels in the environment could control the regulation of the mRNAs by NMD.

COX17 mRNA has been studied extensively and shown to be regulated by puf 3 protein depending on carbon source (Miller et al. 2014). COX23 and COX19 mRNAs are less well understood. Cox 17p, Cox 19p and Cox 23p are essential for $\mathrm{CcO}$ assembly because they are required for mitochondrial copper utilization. Copper is an essential cofactor in the mitochondrial electron transport chain. We report here that the three mRNAs are differentially regulated by NMD depending on environmental copper levels. The differential regulation of the mRNAs by the pathway may have functional significance to yeast cells.

For example, COX17 mRNA is directly regulated by NMD only in rich media but not under low or high copper conditions. It could be that under adequate copper conditions, NMD maintains $C O X 17 \mathrm{mRNA}$ at the appropriate levels for mitochondrial copper utilization. Under low or high copper conditions, COX17 mRNA levels could be adjusted. Additionally, COX17 mRNA has a short ORF of 210 nucleotides. This may well activate NMD-mediated degradation of the mRNA in rich media because the only other recognizable NMD targeting feature is possible leaky scanning that could promote frameshifting and NMD (Celik et al. 2017). As mentioned above, COX17 mRNA decay is regulated by puf3 protein (Olivas and Parker 2000). puf3 protein binds primarily to nuclear encoded mRNAs that encode proteins with mitochondrial function. Thus, in rich media $\mathrm{COX} 17$ mRNA is regulated by puf3 protein and the NMD pathway. Interestingly of the mRNAs investigated here, COX17 and COX23 mRNAs were found to associate with puf3 protein and COX19 mRNA has not been reported to be regulated by puf3 (Gerber et al. 2004; Foat et al. 2005).

On the other hand, the main COX19 mRNA isoform was found to be a direct NMD target under all of conditions tested. The half-life of COX19 mRNA was almost twice as long in wild-type yeast strains grown in rich media relative to low copper or excess copper conditions. This observation suggests that additional COX19 mRNA might be required to translate more Cox 19p to support the metallation of $\mathrm{CcO}$ in 
Fig. 6 The $C O X 23$ 3'-UTR is sufficient to target $C Y C 1 \mathrm{mRNA}$ to NMD. Schematic representation of CYC1COX23 3'-UTR mRNA (a) and representative northern blots of the $\mathrm{CYC}$ 1COX23 3'-UTR steady-state mRNA accumulation levels (b) and half-lives (c). The northern blots were probed with DNA specific to the 5'-UTR and ORF of $C Y C 1$. All yeast cells for $\mathbf{b}$ and $\mathbf{c}$ were grown in synthetic complete media lacking leucine and were harvested as described in Fig. 1. Steady-state and half-life mRNA measurements are an average of at least three independent experiments. The half-lives were determined using SigmaPlot. $\mathrm{CYH} 2$ and $S C R 1$ are controls
A

CYC1COX23 3'-UTR mRNA

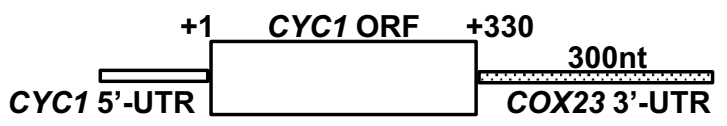

B

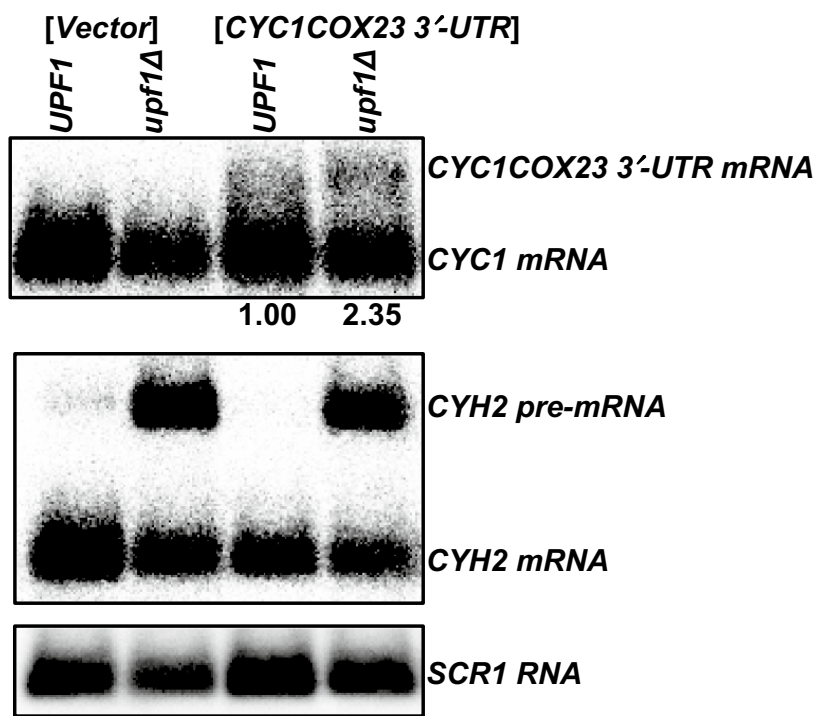

C

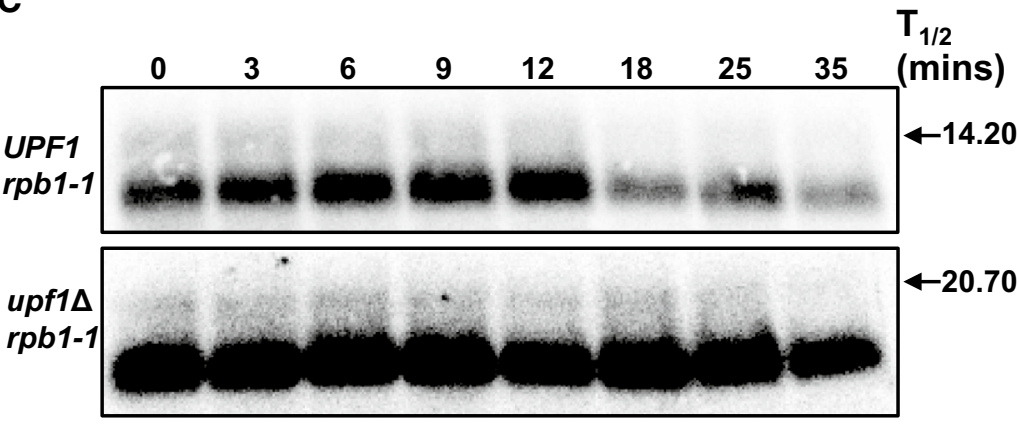

rich media. Interestingly, overexpression of $\mathrm{COX} 19$ resulted in wild-type yeast cells that are more tolerant of toxic copper levels. This observation suggests that precise regulation of COX19 mRNA by NMD under diverse conditions is required to maintain precise mitochondrial copper homeostatic mechanisms. In addition, COX19 mRNA has a long 3'-UTR that contributes to the degradation of the mRNA by NMD (Peccarelli et al. 2016).

Interestingly both $C O X 19$ and COX23 mRNA have identical NMD targeting features but are differentially regulated by NMD. COX23 mRNA is immune to NMD under low copper conditions and is an indirect NMD target in rich media and high copper. However, COX23 mRNA accumulates to higher levels in these conditions; this indicates that there are other factors upstream of COX 23 mRNA that regulate the mRNA levels in response to NMD. This suggests that the NMD pathway, based on environmental conditions, may differentially regulate homologous mRNAs with identical NMD targeting features. Furthermore, because the COX19 mRNA $3^{\prime}$-UTR is twice as long as the COX23 mRNA3'UTR, it could be that the COX19 $3^{\prime}$-UTR is a more efficient NMD targeting feature compared to the COX23 3'-UTR.

However, we found that the COX23 3 '-UTR is sufficient to target an NMD-insensitive mRNA to the pathway. This observation shows that the COX23 mRNAs 3'-UTR is not too short to directly target the mRNA to the NMD pathway. It is possible that the endogenous COX23 mRNA's 3'-UTR is in the incorrect context or that the mRNA is directly regulated by NMD in specific environmental conditions. As mentioned above, we previously found that an additional mRNA belonging to this general functional group, MACl mRNA, was directly regulated by NMD in rich media but not under 
A

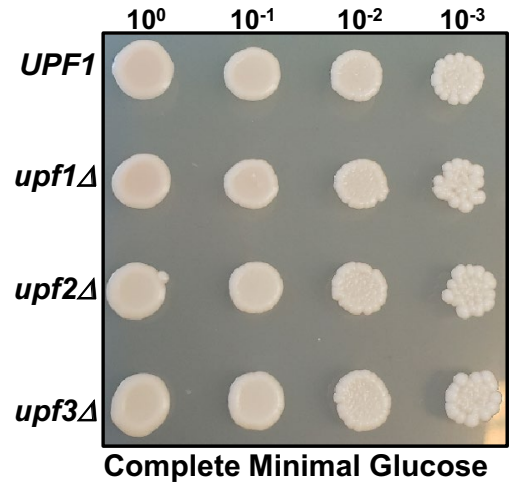

B

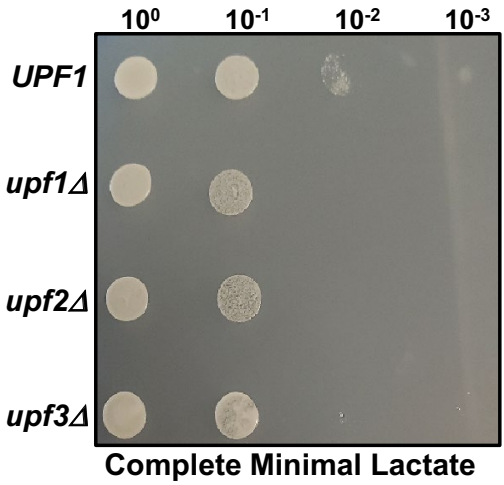

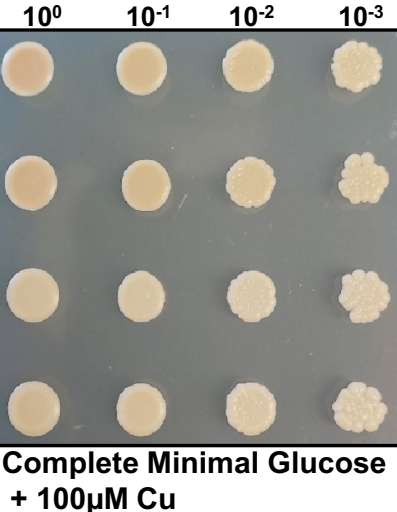
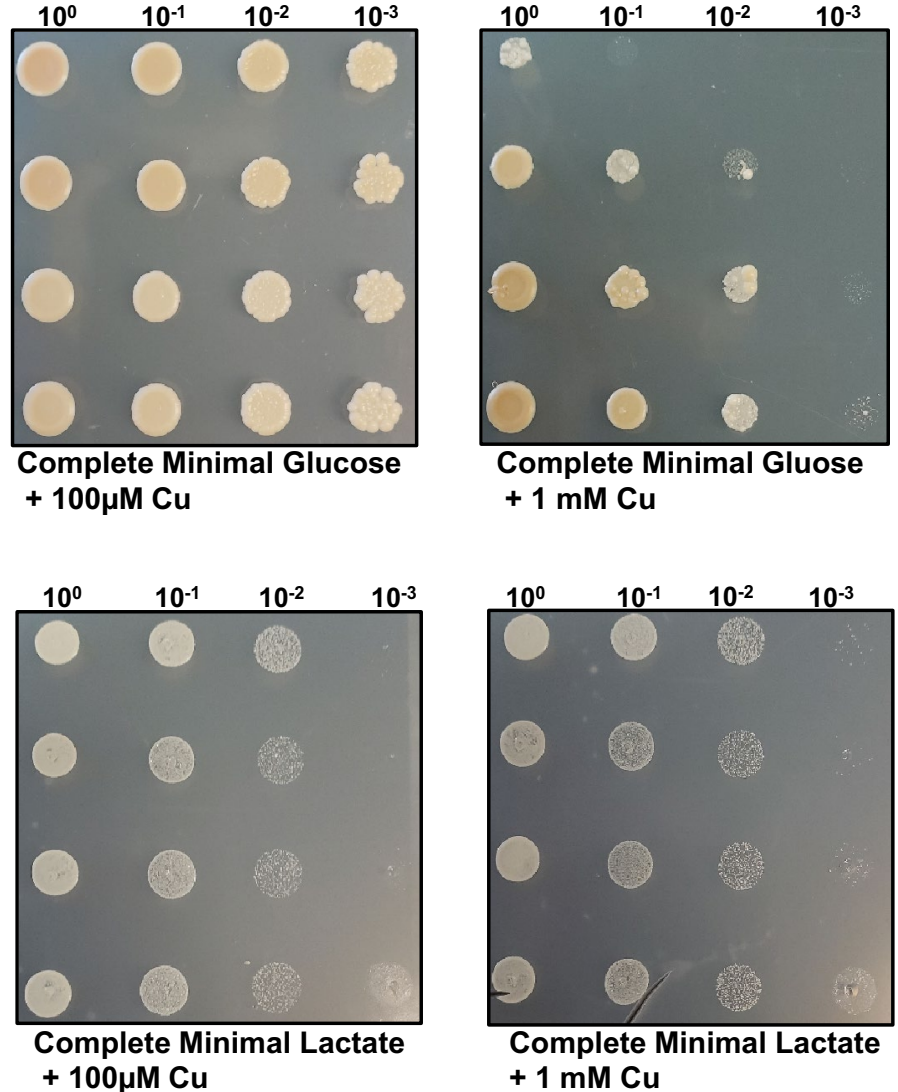

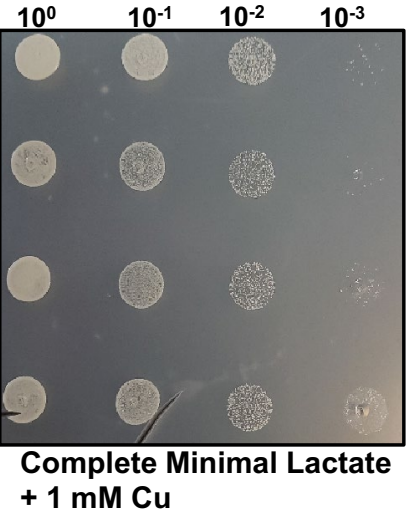

Fig. 7 NMD mutants' respiratory impairments on non-fermentable carbon sources is recovered by elevated copper levels. UPF1

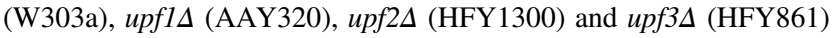
yeast cells were grown to mid-log phase on complete minimal media. Tenfold serial dilutions of the cells were spotted onto complete

low copper conditions (Peccarelli et al. 2016). We hypothesize that a feature within the 5'-UTR or ORF of COX23 can stabilize the transcript. Alternatively, factors present in specific conditions can bind to the COX23 mRNA and stabilize it. Further investigation into how COX23 mRNA evades direct regulation by NMD in rich media, and high copper and the extent to which additional environmental conditions lead to direct NMD targeting of COX23 mRNA will provide insights into the targeting of functional groups of mRNAs.

Regulation of mRNAs involved in mitochondrial copper utilization by NMD has physiological consequences to yeast cells. Expression of Cox17, Cox 19 and Cox 23 proteins are required for respiratory capability (Longen et al. 2009). Furthermore, NMD mutants have impaired growth on non-fermentable carbon sources. Additional copper in the growth media can enhance the impaired growth defect of the NMD mutants. This suggests that imbalance in mitochondrial copper homeostasis is partly responsible for the NMD mutants' respiratory defect. Interestingly, additional copper can recover impaired growth of a COX17 mutant minimal medium with either glucose (a) or lactate (b) as the carbon source. Additionally, the four yeast strains were spotted on media containing either $100 \mu \mathrm{M}$ copper (middle panels) or $1 \mathrm{mM}$ copper (right panels) and incubated $30^{\circ} \mathrm{C}$ for 3 days

but not a COX19 mutant. Additionally, extra copper can also enhance the growth of NMD mutants grown on nonfermentable carbon sources. This observation suggests that misregulation of mitochondrial copper homeostatic mRNAs is partly responsible for the growth defect. This study adds to reports demonstrating that mitochondrial gene expression is regulated at the RNA level. Specifically long noncoding RNAs have been reported to regulate mitochondrial gene expression (De Paepe et al. 2018). In summary, the studies reported here show mitochondrial copper homeostatic mechanisms occur at the mRNA level via the NMD pathway.

\section{Conclusions}

In this study, we showed that: (1) COX17 mRNA is a direct NMD target under specific growth conditions; (2) COX17, COX19 and COX23 mRNAs are differentially regulated by NMD based on environmental copper levels; (3) we 
A Cox17 overexpression

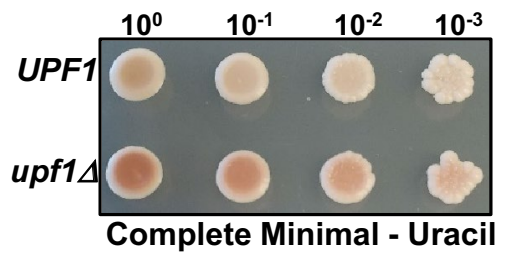

B Cox19 overexpression

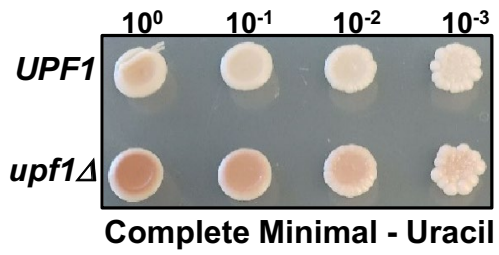

C Cox23 overexpression

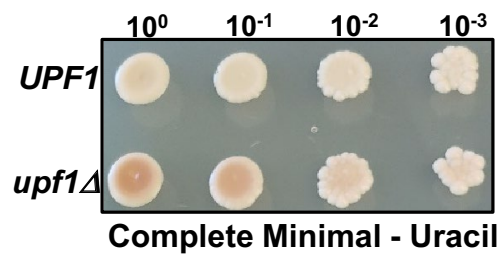

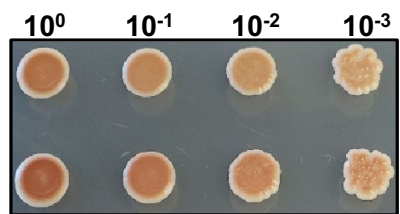

Complete Minimal - Uracil $+100 \mu \mathrm{M} \mathrm{Cu}$

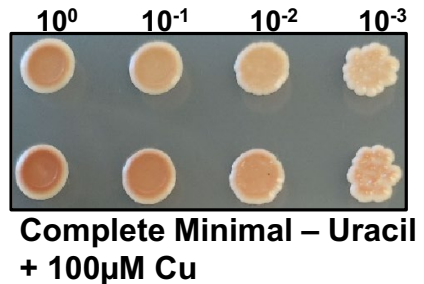

$+100 \mu \mathrm{M} \mathrm{Cu}$

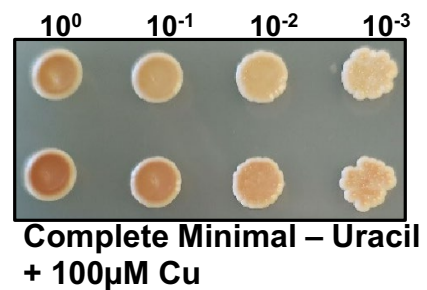

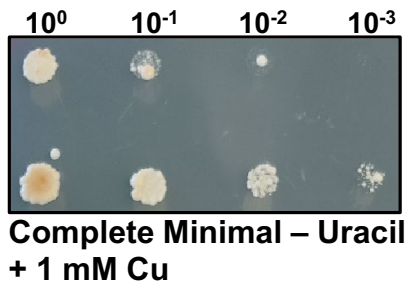

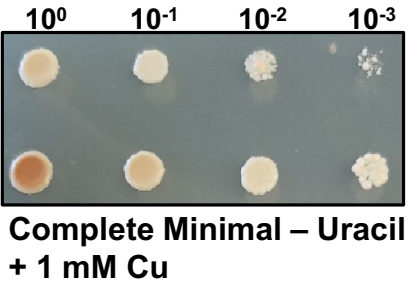

$+1 \mathrm{mM} \mathrm{Cu}$

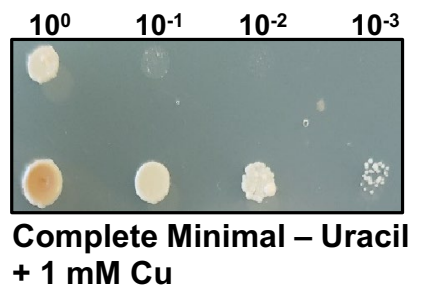

Fig. 8 Overexpression of $C O X 19$ enhances growth of wild-type yeast strains on media containing elevated copper levels. UPF1 (W303a) and upf1 $\Delta$ (AAY327) yeast strain were transformed with pGT74/T3 (a), pG188/T1 (b) and pG84/T1 (c) plasmids and grown to mid-log

determined that the COX23 3 '-UTR is sufficient to trigger NMD; (4) growth impairment of wild-type and NMD mutants on non-fermentable carbon source is enhanced by excess copper; (5) overexpression of COX19 enhances growth of wild-type yeast strains on toxic amounts of copper. This study as well as our previous studies suggests that regulation of functionally related mRNAs by NMD may be dependent on environmental conditions and can be differential. Our study also provides insight into the mechanism NMD plays in regulating natural mRNAs.

Acknowledgements We would like to thank Dr. Alexander Tzagoloff for plasmids and yeast strains. Additionally, we thank the Molecular Biosciences Center (MBC) facility at Baylor University for equipment and supplies.

Author contributions KM, MH, MP and TS performed the experiments, analyzed the data and prepared the manuscript. BK planned the experiments, contributed to the preparation of the manuscript and approved the final manuscript.

Funding This research reported in this publication was supported by grants from the Texas Higher Education Coordinating Board's Norman Hackerman Advanced Research Program and by the National Institute phase on complete minimal media lacking uracil. Tenfold serial dilutions of the cells were spotted onto complete minimal medium lacking uracil (left panels), containing either $100 \mu \mathrm{M}$ copper (middle panels) or $1 \mathrm{mM}$ copper (right panels) and incubated $30^{\circ} \mathrm{C}$ for 4 days

of General Medical Sciences of the National Institutes of Health under Award Number R15GM117524. The content is solely the responsibility of the authors and does not necessarily represent the official views of the National Institutes of Health.

\section{Compliance with ethical standards}

Ethical statement The research presented in this manuscript has not been submitted previously or concurrently to another journal, does not contain any fabricated or manipulated data and has not been split into several parts. The contributions of each of the author listed are detailed above in the authors' contributions.

Open Access This article is distributed under the terms of the Creative Commons Attribution 4.0 International License (http://creativeco mmons.org/licenses/by/4.0/), which permits unrestricted use, distribution, and reproduction in any medium, provided you give appropriate credit to the original author(s) and the source, provide a link to the Creative Commons license, and indicate if changes were made. 


\section{References}

Ausubel FM, Brent R, Kingston RE, Moore DD, Seidman JG, Smith JA, Struhl K (1998) Current protocols in molecular biology. Wiley, New York

Belew AT, Advani VM, Dinman JD (2011) Endogenous ribosomal frameshift signals operate as mRNA destabilizing elements through at least two molecular pathways in yeast. Nucleic Acids Res 39:2799-2808

Celik A, He F, Jacobson A (2017) NMD monitors translational fidelity 24/7. Curr Genet 63:1007-1010

De Freitas J, Wintz H, Kim JH, Poynton H, Fox T, Vulpe C (2003) Yeast, a model organism for iron and copper metabolism studies. Biometals 16:185-197

De Paepe B, Lefever S, Mestdagh P (2018) How long noncoding RNAs enforce their will on mitochondrial activity: regulation of mitochondrial respiration, reactive oxygen species production, apoptosis, and metabolic reprogramming in cancer. Curr Genet 64:163-172

Deliz-Aguirre R, Atkin AL, Kebaara BW (2011) Copper tolerance of Saccharomyces cerevisiae nonsense-mediated mRNA decay mutants. Curr Genet 57:421-430

Foat BC, Houshmandi SS, Olivas WM, Bussemaker HJ (2005) Profiling condition-specific, genome-wide regulation of mRNA stability in yeast. Proc Natl Acad Sci USA 102:17675-17680

Gaba A, Jacobson A, Sachs MS (2005) Ribosome occupancy of the yeast CPA1 upstream open reading frame termination codon modulates nonsense-mediated mRNA decay. Mol Cell 20:449-460

Gerber AP, Herschlag D, Brown PO (2004) Extensive association of functionally and cytotopically related mRNAs with Puf family RNA-binding proteins in yeast. PLoS Biol 2:E79

Guan Q, Zheng W, Tang S, Liu X, Zinkel RA, Tsui KW, Yandell BS, Culbertson MR (2006) Impact of nonsense-mediated mRNA decay on the global expression profile of budding yeast. PLoS Genet 2:e203

Guisbert E, Rhodius VA, Ahuja N, Witkin E, Gross CA (2007) Hfq modulates the sigmaE-mediated envelope stress response and the sigma32-mediated cytoplasmic stress response in Escherichia coli. J Bacteriol 189:1963-1973

He F, Jacobson A (1995) Identification of a novel component of the nonsense-mediated mRNA decay pathway by use of an interacting protein screen. Genes Dev 9:437-454

He F, Peltz SW, Donahue JL, Rosbash M, Jacobson A (1993) Stabilization and ribosome association of unspliced pre-mRNAs in a yeast upf1-mutant. Proc Natl Acad Sci USA 90:7034-7038

He F, Brown AH, Jacobson A (1997) Upf1p, Nmd2p, and Upf3p are interacting components of the yeast nonsense-mediated mRNA decay pathway. Mol Cell Biol 17:1580-1594

He F, Li X, Spatrick P, Casillo R, Dong S, Jacobson A (2003) Genomewide analysis of mRNAs regulated by the nonsense-mediated and $5^{\prime}$ to $3^{\prime}$ mRNA decay pathways in yeast. Mol Cell 12:1439-1452
Johansson MJ, Jacobson A (2010) Nonsense-mediated mRNA decay maintains translational fidelity by limiting magnesium uptake. Genes Dev 24:1491-1495

Kebaara BW, Atkin AL (2009) Long 3'-UTRs target wild-type mRNAs for nonsense-mediated mRNA decay in Saccharomyces cerevisiae. Nucleic Acids Res 37:2771-2778

Kebaara B, Nazarenus T, Taylor R, Atkin AL (2003) Genetic background affects relative nonsense mRNA accumulation in wildtype and upf mutant yeast strains. Curr Genet 43:171-177

Kebaara BW, Baker KE, Patefield KD, Atkin AL (2012) Analysis of nonsense-mediated mRNA decay in Saccharomyces cerevisiae. Curr Protoc Cell Biol Chap 27:Unit 27.23

Longen S, Bien M, Bihlmaier K, Kloeppel C, Kauff F, Hammermeister M, Westermann B, Herrmann JM, Riemer J (2009) Systematic analysis of the twin cx(9)c protein family. J Mol Biol 393:356-368

Mendell JT, Sharifi NA, Meyers JL, Martinez-Murillo F, Dietz HC (2004) Nonsense surveillance regulates expression of diverse classes of mammalian transcripts and mutes genomic noise. Nat Genet 36:1073-1078

Miller MA, Russo J, Fischer AD, Lopez Leban FA, Olivas WM (2014) Carbon source-dependent alteration of Puf3p activity mediates rapid changes in the stabilities of mRNAs involved in mitochondrial function. Nucleic Acids Res 42:3954-3970

Olivas W, Parker R (2000) The Puf3 protein is a transcript-specific regulator of mRNA degradation in yeast. EMBO J 19:6602-6611

Peccarelli M, Kebaara BW (2014) Measurement of mRNA decay rates in Saccharomyces cerevisiae using rpb1-1 strains. J Vis Exp 13:94

Peccarelli M, Scott TD, Wong H, Wang X, Kebaara BW (2014) Regulation of CTR 2 mRNA by the nonsense-mediated mRNA decay pathway. Biochim Biophys Acta 1839:1283-1294

Peccarelli M, Scott TD, Steele M, Kebaara BW (2016) mRNAs involved in copper homeostasis are regulated by the nonsensemediated mRNA decay pathway depending on environmental conditions. Fungal Genet Biol 86:81-90

Rehwinkel J, Letunic I, Raes J, Bork P, Izaurralde E (2005) Nonsensemediated mRNA decay factors act in concert to regulate common mRNA targets. RNA 11:1530-1544

Welch EM, Jacobson A (1999) An internal open reading frame triggers nonsense-mediated decay of the yeast SPT10 mRNA. EMBO J 18:6134-6145

Wente SR, Rout MP, Blobel G (1992) A new family of yeast nuclear pore complex proteins. J Cell Biol 119:705-723

Wittmann J, Hol EM, Jack HM (2006) hUPF2 silencing identifies physiologic substrates of mammalian nonsense-mediated mRNA decay. Mol Cell Biol 26:1272-1287

Zaret KS, Sherman F (1984) Mutationally altered 3' ends of yeast CYC1 mRNA affect transcript stability and translational efficiency. J Mol Biol 177:107-135 